\title{
Image Registration Based on Thin-Plate Splines and Local Estimates of Anisotropic Landmark Localization Uncertainties
}

\author{
Karl Rohr \\ Universität Hamburg, Fachbereich Informatik, AB Kognitive Systeme, \\ Vogt-Kölln-Str. 30, D-22527 Hamburg, Germany \\ rohr@informatik . uni-hamburg. de
}

\begin{abstract}
We present an approach to elastic registration of tomographic brain images which is based on thin-plate splines and takes into account landmark errors. The inclusion of error information is important in clinical applications since landmark extraction is always prone to error. In comparison to previous work, our approach can cope with anisotropic errors, which is significantly more realistic than dealing only with isotropic errors. In particular, it is now possible to include different types of landmarks, e.g., quasi-landmarks at the outer contour of the brain. Also, we introduce an approach to estimate landmark localization uncertainties directly from the image data. Experimental results are presented for the registration of $2 \mathrm{D}$ and $3 \mathrm{D} \mathrm{MR}$ images.
\end{abstract}

\section{Motivation}

Image registration is fundamental to computer-assisted neurosurgery. Examples are the registration of tomographic images and the registration of images with digital atlases. In either case, the central aim is to increase the accuracy of localizing anatomical structures in 3D space. One principal approach to registration is based on thin-plate splines and anatomical point landmarks.

Previous work on thin-plate spline registration has concentrated on specifying the landmarks manually and on using an interpolating transformation model (e.g., [2],[6],[11]). The approach is efficient and well-suited for user-interaction which we consider important in clinical scenaria. However, with an interpolation scheme the corresponding landmarks are forced to match exactly. It is (implicitly) assumed that the landmark positions are known exactly, which, however, is generally not true in practical applications. Therefore, to take into account landmark localization errors, we have recently introduced an approximation scheme ([13]). This scheme is based on a minimizing functional, it uses scalar weights to represent landmark errors, and it has been described for images of arbitrary dimensions. The applicability of our approach has been demonstrated for 2D MR images. Bookstein [3] has proposed a different approach to relax the interpolation conditions. His approach, however, has not been related to a minimizing 
functional, but combines different metrics based on a technique called 'curve décolletage'. Also, the approach has only been described for 2D datasets, and experimental results have been reported for 2D synthetic data ('simulated PET images').

One problem with our approach in [13] is that scalar weights for the landmarks are only a coarse characterization of the localization errors. Generally, the errors are different in different directions and thus are anisotropic. Another problem is how to specify the landmark errors.

In this contribution, we describe an extension of our previous approach [13], which allows to incorporate full covariance matrices representing landmark position errors. Although the corresponding minimizing functional is more complicated than in the case of using only scalar weights, we still have an analytic solution the parameters of which can efficiently be computed by solving an analogous linear system of equations. Also, we introduce an approach to estimate the covariance matrices directly from the image data. In our case, these matrices represent the minimal localization uncertainty at landmark points (Cramér-Rao bound). An advantage of our approach is that we can now include different types of 3D point landmarks, e.g., 'normal' point landmarks as well as 'quasilandmarks'. Quasi-landmarks are not uniquely definable in all directions (e.g., arbitrary edge points) and they are used, for example, in the reference system of Talairach [15] to define the 3D bounding box of the brain. The incorporation of such landmarks is important since 'normal' point landmarks are hard to define, for example, at the outer parts of the brain.

The remainder of this contribution is organized as follows. In the next section, we provide a statistical interpretation of our approach to landmark localization. This interpretation is also the basis for estimating landmark localization uncertainties directly from the image data as described in Section 3 . Section 4 is then devoted to the thin-plate spline approach using weight matrices, and Section 5 demonstrates the applicability of our approach for $2 \mathrm{D}$ as well as $3 \mathrm{D}$ tomographic images of the human brain.

\section{Statistical Interpretation of 3D Landmark Localization}

3D anatomical point landmarks are usually localized manually. Generally, this procedure is difficult, time-consuming, and often lacks accuracy (e.g., [6],[9]). To improve on this, we use a semi-automatic procedure with the advantage that the user has the possibility to control the results (see also [13]). Semi-automatic means that landmark candidates are automatically detected within a specified region-of-interest (ROI) and then the user selects the most promising candidate. To simplify the selection, the landmark candidates are ordered based on the automatically computed operator responses. The anatomical landmarks we use are located on the skull base (e.g., tip of external protuberance, saddle point on zygomatic bone) as well as on the ventricular system (e.g., tips of frontal and occipital horns). 
To extract anatomical point landmarks, we here consider a 3D extension of a $2 \mathrm{D}$ corner operator. In previous work $([13],[12])$ this approach has been motivated by the criterion: 'Find points with high intensity variations'. This criterion, however, is rather unspecific. In this contribution, we show that a statistical interpretation is possible. We consider the $3 \mathrm{D}$ differential operator

$$
\operatorname{det} \mathbf{C}_{g} \rightarrow \max ,
$$

where $\mathbf{C}_{g}=\overline{\nabla g(\nabla g)^{T}}$ is a symmetric $3 \times 3$ matrix which represents the averaged dyadic product of the 3D intensity gradient $\nabla g=\left(g_{x}, g_{y}, g_{z}\right)^{T}, g(x, y, z)$ is the image function, and det denotes the determinant of a matrix. Note, that the operator in (1) requires to compute only first order partial derivatives of an image. Therefore, the operator is computationally efficient and does not suffer from instabilities of computing high order partial derivatives. In contrast to that, other 3D differential operators require partial derivatives up to order two or three (e.g., [17],[10]).

In the following, let $\sigma_{n}^{2}$ denote the variance of additive white Gaussian image noise and $m$ the number of voxels in a local 3D window. Then, we can relate the matrix $\mathbf{C}_{g}$ to the minimal localization uncertainty of the center of the window $\mathbf{x}=(x, y, z)$. The minimal localization uncertainty is given by the Cramér-Rao bound (e.g., [18]) and is represented by the covariance matrix

$$
\Sigma_{g}=\frac{\sigma_{n}^{2}}{m} \mathbf{C}_{g}^{-1}
$$

We see, that $\boldsymbol{\Sigma}_{g}$ is proportional to the inverse of $\mathbf{C}_{g}$. From (2) we can derive the 3D error ellipsoid of the position estimate with semi-axes $\sigma_{x}, \sigma_{y}$, and $\sigma_{z}$. A quantitative measure for the localization uncertainty of a landmark is the volume of the $3 \mathrm{D}$ error ellipsoid which is defined as

$$
V=\frac{4}{3} \pi \sqrt{\operatorname{det} \Sigma_{g}}
$$

where $\operatorname{det} \boldsymbol{\Sigma}_{g}=\sigma_{x}^{2} \sigma_{y}^{2} \sigma_{z}^{2}$. The smaller $\operatorname{det} \boldsymbol{\Sigma}_{g}$ the smaller is the localization uncertainty. Thus, we can formulate the following criterion for localizing $3 \mathrm{D}$ point landmarks: 'Find points with minimal localization uncertainty', i.e., minimal volume of the $3 \mathrm{D}$ error ellipsoid. This requirement can be stated as

$$
\operatorname{det} \boldsymbol{\Sigma}_{g} \rightarrow \min
$$

Since $\operatorname{det} \Sigma_{g}=1 / \operatorname{det} \Sigma_{g}^{-1}$ and with (2) we see that (4) is equivalent to the operator in (1). Thus, this operator extracts 3D points with minimal localization uncertainty. Note, that in the case we have no Gaussian image noise, we consider (2) as an approximation. Note also, that $\mathbf{C}_{g}$ and thus $\boldsymbol{\Sigma}_{g}$ can directly be computed from the image data, and that $\operatorname{det} \mathbf{C}_{g}$ is invariant w.r.t. translations and rotations. 


\section{Estimation of Landmark Localization Uncertainties}

Our aim is to integrate landmark errors in elastic registration of $3 \mathrm{D}$ images, and in the general case we have to deal with anisotropic errors. But how should we specify these errors? One possibility is to exploit error information from anatomical studies concerning the variability of brain structures. However, currently we are not aware that such anatomical knowledge is available. Alternatively, a user in a certain registration application may provide confidence levels of how well localization of single landmarks is possible (e.g., high, middle, low confidence) as well as may specify certain directions where the uncertainty is extremal. A disadvantage of this procedure is, however, that it is time-consuming.

Instead, we here propose a different approach which allows to estimate landmark localization uncertainties directly from the image data. With this approach we exploit the minimal stochastic error due to the intensity variations in the neighborhood of a landmark. The minimal error is determined by the CramérRao bound stated in (2). This bound depends on the local variation of the image gradient as well as on the image noise. Note, that since we use a lower bound this procedure is applicable to any point within an image, independently of how this point has been localized (manually or automatically). The required first order image derivatives in (2) in our case are computed by applying Gaussian derivative filters or Beaudet filters. Averaging is generally performed in a $5 \times 5 \times 5$ neighborhood.

Based on (2) we can distinguish different types of 3D landmark points. Landmarks with locally high intensity variations in all directions have low localization uncertainties in all directions and we refer to them as 'normal' point landmarks. Arbitrary points on image edges have low localization uncertainties perpendicular to the edge but high localization uncertainties along the edge. Such landmarks will be denoted as 'quasi-landmarks' since they are not uniquely definable in all directions (e.g., the 3D bounding box landmarks of Talairach [15]). Finally, normal landmarks and quasi-landmarks can be distinguished from points in homogeneous regions where we have high localization uncertainties in all directions (see [7] for an analogous classification in the case of 2D aerial images).

Note, that our error characterization is based on the minimal errors, which are generally different from the errors obtained by an anatomical study or specified by a user. However, in either case we suspect that the localization errors depend on the geometry of the landmark, e.g., for an edge point we have large uncertainties along the edge but low uncertainties perpendicular to the edge. Also, all approaches should share the property that increasing the image noise generally leads to larger localization errors, which is the case with our scheme.

As an example, we show in Fig. 1 the estimated error ellipses (68.3\% probability corresponding to $1 \sigma$ regions) for the tip of the frontal horn of the ventricular system as well as for an arbitrary edge point at the outer contour of the head within a 2D MR dataset. The selected points are examples for normal landmarks and quasi-landmarks. Note, that the error ellipses have been enlarged by a factor of 30 for visualization purposes. It can be seen that the error ellipse of the tip is small and close to a circle which means that the localization uncertainty for this 

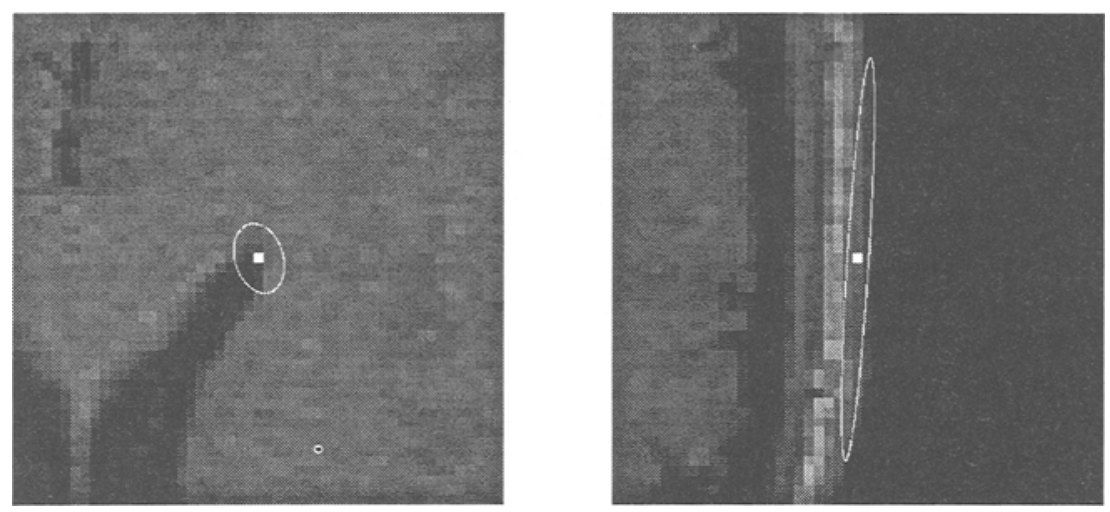

Fig. 1. Estimated 2D error ellipses (enlarged by a factor of 30) for a 2D MR dataset: tip of ventricular system (left) and edge point at the outer contour of the head (right).

point is low in arbitrary directions. For the edge point, however, the error ellipse is largely elongated and indicates a large localization uncertainty along the contour and a low localization uncertainty perpendicular to the contour. This is what we expect from the local intensity structure at the considered points.

\section{Thin-Plate Splines with Anisotropic Errors}

In [13] we have introduced an approach for nonrigid matching of medical images using approximating thin-plate splines. This approach is based on the mathematical work in [19] and is an extension of the original interpolating thin-plate spline approach [2]. For related approaches in the context of surface approximation and modification of facial expressions, see, e.g., [8],[5],[16],[1].

To find the transformation $\mathbf{u}$ between two images of dimension $d$ we assume to have two sets of $n$ landmarks $\mathbf{p}_{i}$ and $\mathbf{q}_{i}, i=1 \ldots n$, in the first and second image, resp., as well as information about the landmark localization errors in terms of scalar weights $\sigma_{i}^{2}$. Then, $\mathbf{u}$ results as the solution of a minimizing functional which measures the distance between the two landmark sets and the smoothness of the transformation:

$$
J_{\lambda}(\mathbf{u})=\frac{1}{n} \sum_{i=1}^{n} \frac{\left|\mathbf{q}_{i}-\mathbf{u}\left(\mathbf{p}_{i}\right)\right|^{2}}{\sigma_{i}^{2}}+\lambda J_{m}^{d}(\mathbf{u}),
$$

where $m$ is the order of the involved derivatives of $\mathbf{u}$, and the parameter $\lambda>0$ weights the two terms. This functional can be separated into a sum of functionals which only depend on one component of $\mathbf{u}$. The solution to the single functionals can be stated analytically as

$$
u(\mathbf{x})=\sum_{\nu=1}^{M} a_{\nu} \phi_{\nu}(\mathbf{x})+\sum_{i=1}^{n} w_{i} U\left(\mathbf{x}, \mathbf{p}_{i}\right)
$$


with polynomials $\phi$ up to order $m-1$ and suitable radial basis functions $U$ as defined in [19]. For example, in the case $m=d=2$ we have the well-known function $U(\mathbf{x}, \mathbf{p})=1 /(8 \pi)|\mathbf{x}-\mathbf{p}|^{2} \ln |\mathbf{x}-\mathbf{p}|$.

The coefficients $\mathbf{a}=\left(a_{1}, \ldots, a_{M}\right)^{T}$ and $\mathbf{w}=\left(w_{1}, \ldots, w_{n}\right)^{T}$ of the transformation can be computed through the following system of linear equations:

$$
\begin{aligned}
\left(\mathbf{K}+n \lambda \mathbf{W}^{-\mathbf{1}}\right) \mathbf{w}+\mathbf{P a} & =\mathbf{v} \\
\mathbf{P}^{T} \mathbf{w} & =0
\end{aligned}
$$

where $\mathbf{v}$ is a vector of one component of the coordinates of the landmarks $\mathbf{q}_{i}$, $K_{i j}=U\left(\mathbf{p}_{i}, \mathbf{p}_{j}\right)$, and $P_{i j}=\phi_{j}\left(\mathbf{p}_{i}\right)$. The matrix

$$
\mathbf{W}^{-1}=\operatorname{diag}\left\{\sigma_{1}^{2}, \ldots, \sigma_{n}^{2}\right\}
$$

contains the scalar weights representing isotropic landmark localization errors.

This approach can further be extended when replacing the scalar weights $\sigma_{i}^{2}$ by covariance matrices $\Sigma_{i}$ representing anisotropic landmark errors. With $\epsilon_{i}=\mathbf{q}_{i}-\mathbf{u}\left(\mathbf{p}_{i}\right)$ now our functional reads as

$$
J_{\lambda}(\mathbf{u})=\frac{1}{n} \sum_{i=1}^{n} \epsilon_{i}^{T} \Sigma_{i}^{-1} \epsilon_{i}+\lambda J_{m}^{d}(\mathbf{u})
$$

Although this generalized functional can no longer be separated into the components of $\mathbf{u}$, the solution can still be stated in analytic form with the same basis functions and the same structure of the computational scheme as in (7), see also $[20],[14]$. In our case, the weight matrix in (7) is given by

$$
\mathbf{W}^{-1}=\operatorname{diag}\left\{\boldsymbol{\Sigma}_{1}, \ldots, \boldsymbol{\Sigma}_{n}\right\}
$$

and is a block-diagonal matrix. Note, that the $\boldsymbol{\Sigma}_{i}$ represent the localization errors of two corresponding landmarks. Thus, to end up with one matrix we have to combine the covariance matrices of corresponding landmarks. If we assume that the two covariance matrices depend only slightly on the elastic part of the transformation, then we can combine these matrices by applying a linear transformation (rotation, scaling) to one of the matrices before adding them. The other matrices in (7) are given by $\mathbf{K}=\left(K_{i j} \mathbf{I}_{d}\right)$, where $K_{i j}=U\left(\mathbf{p}_{i}, \mathbf{p}_{j}\right)$ and $\mathbf{I}_{d}$ is the $d \times d$ unity matrix, and $\mathbf{P}=\left(P_{i j} \mathbf{I}_{d}\right)$, where $P_{i j}=\phi_{j}\left(\mathbf{p}_{i}\right)$.

With the extended registration scheme it is now possible to include 'quasilandmarks'. Note, that our approximation scheme using weight matrices is also a generalization of the work in [4], where the interpolation problem is solved while the landmarks are allowed to slip along straight lines within a 2D image. Actually, this is a special case of our approximation scheme since for straight lines the variance in one direction is zero whereas in the perpendicular direction it is infinity. 


\section{Experimental Results}

\subsection{D Data}

We have applied our approach to the registration of $2 \mathrm{D} M R$ brain images of different patients shown in Fig. 2. In this 2D example we have manually selected normal landmarks and quasi-landmarks and have automatically estimated the error ellipses at these points (note, that the ellipses drawn in Fig. 2 have been enlarged by a factor of 7 for visualization purposes). Fig. 3 on the left shows the registration result when applying interpolating thin-plate splines while using all landmarks. The edges of the second image have been overlayed onto the transformed first image. We see that an unrealistic deformation towards the top left is obtained since corresponding landmarks are forced to match exactly although they are generally not homologous points. Using instead approximating thin-plate splines and equal scalar weights $(m=d=2$ in (5)) the registration result is improved but it is still not satisfactory (Fig. 3 on the right). A further improvement is obtained if we apply this procedure only to the normal landmarks (numbers 1-6 and 8) in the inner parts of the brain (Fig. 4 on the left). However, the best result is achieved if we use both the normal landmarks and the quasilandmarks together with the estimated ellipses, and apply approximating thinplate splines with anisotropic errors (Fig. 4 on the right). It can be seen that the combination of both types of landmarks significantly improves the registration accuracy, particularly at the outer contour of the brain.

\subsection{D Data}

We also demonstrate the applicability of our approach for the registration of 3D MR images of different patients. The datasets consist of $179 \times 236 \times 165$ and $177 \times 245 \times 114$ voxels, resp. (see Fig. 5). We have used normal landmarks as well as quasi-landmarks while setting $m=2$ and $d=3$ in (9). The normal point landmarks have semi-automatically been localized using the 3D differential operator in (1). As quasi-landmarks we have used the 3D bounding box landmarks of the brain (Talairach [15]) as well as two landmarks at the top of the ventricular system. The quasi-landmarks have been localized manually. For all landmarks the weight matrices have automatically been estimated from the image data according to (2). Fig. 6 shows that we generally obtain a good registration result (see slices 29, 41, and 67), while some deviations can be observed at the bottom left of the slices 41 and 67 .

\section{Conclusion}

We have introduced a thin-plate spline approach to elastic image registration which can cope with anisotropic landmark errors. The covariance matrices of landmark errors are directly estimated from the image data and represent the 

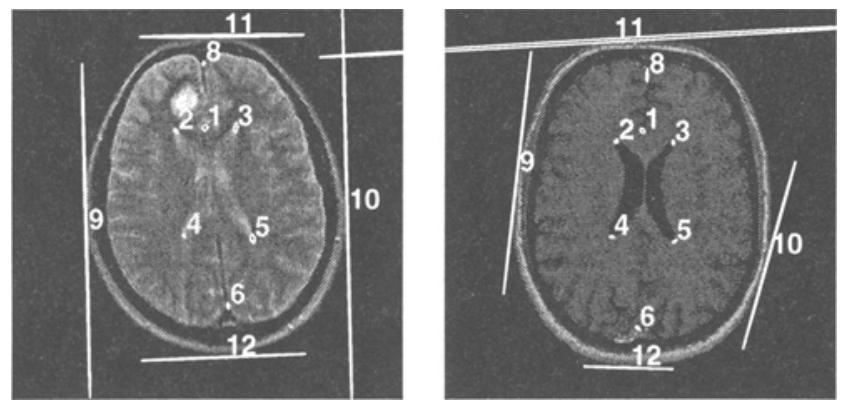

Fig. 2. 2D MR datasets of different patients: normal landmarks, quasi-landmarks, and estimated error ellipses (enlarged by a factor of 7 ).
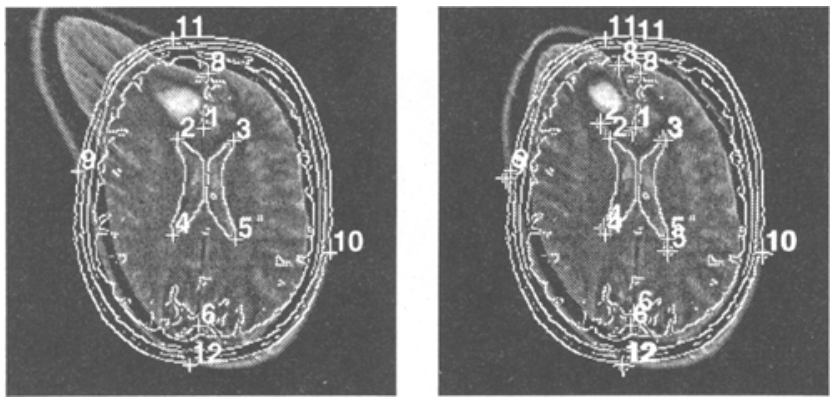

Fig. 3. Registration result: Thin-plate spline interpolation (left), and approximation with equal scalar weights (right) using normal landmarks and quasi-landmarks.
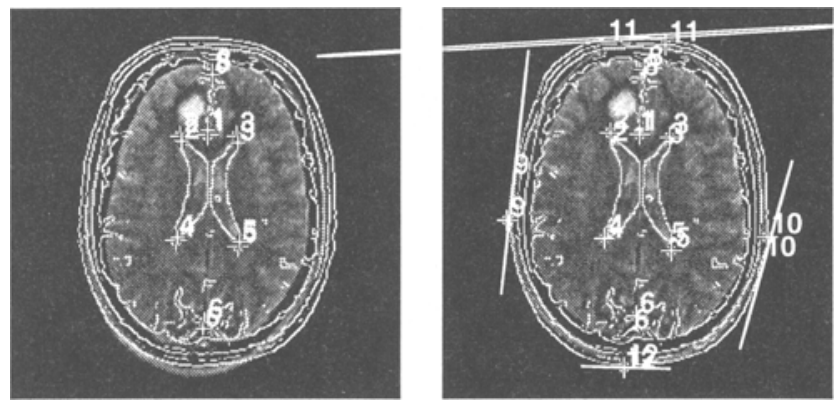

Fig. 4. Registration result: Thin-plate spline approximation using normal landmarks and equal scalar weights (left), and normal landmarks, quasi-landmarks and estimated error ellipses (right).

minimal stochastic localization uncertainties. Besides the handling of error information an additional advantage is that we can include different types of landmarks, e.g., quasi-landmarks. Our experiments for 2D and 3D tomographic images of the human brain have shown that the incorporation of quasi-landmarks significantly improves the registration result. 

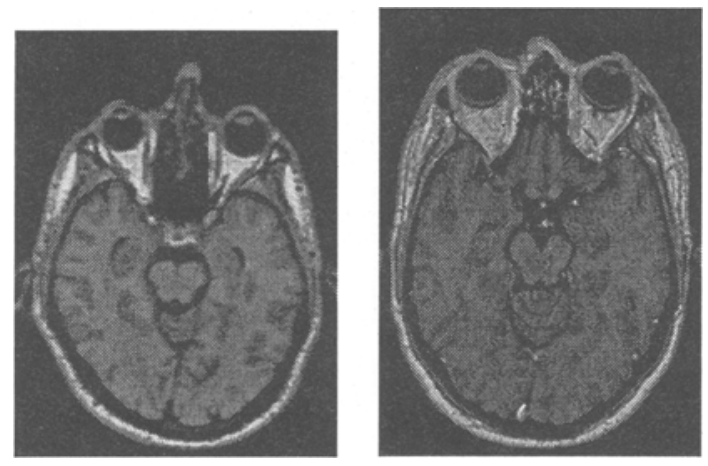

Fig. 5. 3D MR datasets of different patients (slices 57 and 41, resp.).
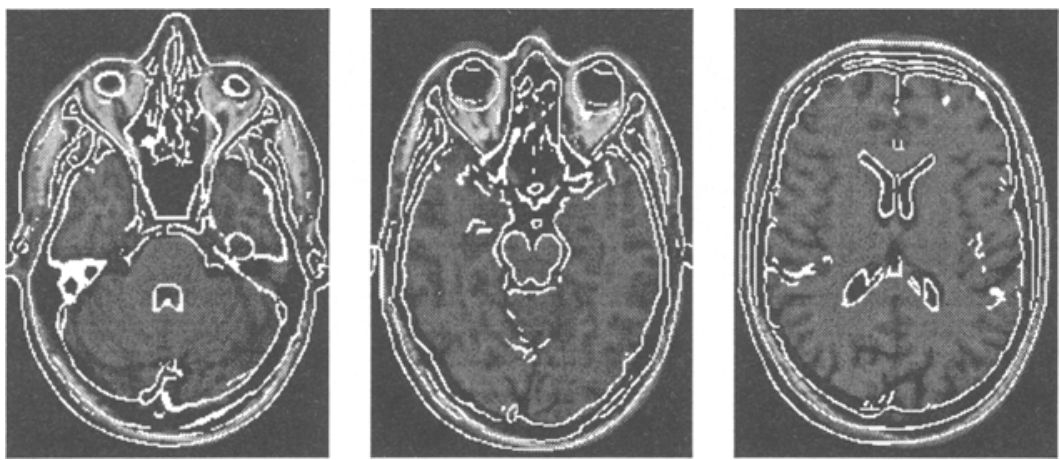

Fig. 6. 3D registration result using approximating thin-plate splines, normal landmarks, quasi-landmarks and estimated $3 \mathrm{D}$ weight matrices (slices 29, 41, and 67).

\section{Acknowledgement}

This work has been supported by Philips Research Hamburg, project IMAGINE. For discussions I thank S. Frantz, R. Sprengel, and H.S. Stiehl. The tomographic images have kindly been provided by Philips Research Hamburg, the AIM project COVIRA as well as W.P.Th.M. Mali, L. Ramos, and C.W.M. van Veelen (Utrecht University Hospital) via ICS-AD of Philips Medical Systems Best.

\section{References}

1. N. Arad, N. Dyn, D. Reisfeld, and Y. Yeshurun, "Image warping by radial basis functions: Application to facial expressions", Computer Vision, Graphics, and Image Processing 56:2 (1994) 161-172

2. F.L. Bookstein, "Principal Warps: Thin-Plate Splines and the Decomposition of Deformations", IEEE Trans. on Pattern Anal. and Machine Intell. 11:6 (1989) 567-585 
3. F.L. Bookstein, "Four metrics for image variation", Proc. IPMI'89, In Progress in Clinical and Biological Research, Vol. 363, D. Ortendahl and J. Llacer (Eds.), Wiley-Liss New York, 1991, 227-240

4. F.L. Bookstein, "Landmark methods for forms without landmarks: morphometrics of group differences in outline shape", Medical Image Analysis 1:3 (1996/7) 225-243

5. T.E. Boult and J.R. Kender, "Visual surface reconstruction using sparse depth data", Proc. CVPR'86, June 22-26, Miami Beach, FL, 1986, 68-76

6. A.C. Evans, W. Dai, L. Collins, P. Neelin, and S. Marrett, "Warping of a computerized 3-D atlas to match brain image volumes for quantitative neuroanatomical and functional analysis", Medical Imaging V: Image Processing, 1991, San Jose, CA, Proc. SPIE 1445, M.H. Loew (Ed.), 236-246

7. W. Förstner, "A Feature Based Correspondence Algorithm for Image Matching", Intern. Arch. of Photogrammetry and Remote Sensing 26-3/3 (1986) 150-166

8. W.E.L. Grimson, "An Implementation of a Computational Theory of Visual Surface Interpolation", Computer Vision, Graphics, and Image Processing 22 (1983) 39-69

9. D.L.G. Hill, D.J. Hawkes, J.E. Crossman, M.J. Gleeson, T.C.S. Cox, E.E.C.M. Bracey, A.J. Strong, and P. Graves, "Registration of MR and CT images for skull base surgery using point-like anatomical features", The British J. of Radiology 64:767 (1991) 1030-1035

10. J.B.A. Maintz, P.A. van den Elsen, and M.A. Viergever, "Evaluation of ridge seeking operators for multimodality medical image matching", IEEE Trans. on Pattern Anal. and Machine Intell. 18:4 (1996) 353-365

11. K. Mardia and J. Little, "Image warping using derivative information", In Mathematical Methods in Medical Imaging III, 25-26 July 1994, San Diego, CA, Proc. SPIE 2299, F.L. Bookstein, J. Duncan, N. Lange, and D. Wilson (Eds.), 16-31

12. K. Rohr, "On 3D Differential Operators for Detecting Point Landmarks", Image and Vision Computing 15:3 (1997) 219-233

13. K. Rohr, H.S. Stiehl, R. Sprengel, W. Beil, T.M. Buzug, J. Weese, and M.H. Kuhn, "Point-Based Elastic Registration of Medical Image Data Using Approximating Thin-Plate Splines", Proc. VBC'96, Hamburg, Germany, Sept. 22-25, 1996, Lecture Notes in Computer Science 1131, K.H. Höhne and R. Kikinis (Eds.), Springer Berlin Heidelberg 1996, 297-306

14. K. Rohr, R. Sprengel, and H.S. Stiehl, "Incorporation of Landmark Error Ellipsoids for Image Registration based on Approximating Thin-Plate Splines", Proc. CAR'97, Berlin, Germany, June 25-28, 1997, H.U. Lemke, M.W. Vannier, and K. Inamura (Eds.), Elsevier Amsterdam Lausanne 1997, 234-239

15. J. Talairach and P. Tornoux, Co-planar Stereotactic Atlas of the Human Brain, Georg Thieme Verlag Stuttgart New York 1988

16. D. Terzopoulos, "Regularization of Inverse Visual Problems Involving Discontinuities", IEEE Trans. on Pattern Anal. and Machine Intell. 8:4 (1986) 413-424

17. J.-P. Thirion, "New Feature Points based on Geometric Invariants for 3D Image Registration", Intern. J. of Computer Vision 18:2 (1996) 121-137

18. H.L. van Trees, Detection, Estimation, and Modulation Theory, Part I, John Wiley and Sons, New York London 1968

19. G. Wahba, Spline Models for Observational Data, Society for Industrial and Applied Mathematics, Philadelphia, Pennsylvania, 1990

20. G. Wahba, "Multivariate function and operator estimation, based on smoothing splines and reproducing kernels", in Nonlinear Modeling and Forecasting, SFI Studies in the Sciences of Complexity, Vol. XII, M. Casdagli and S. Eubank (Eds.), Addison-Wesley 1992, 95-112 